\title{
Auditory Phenotypic Variability in Friedreich's Ataxia Patients
}

\author{
Nehzat Koohi $^{1,2,3}$ (D) $\cdot$ Gilbert Thomas-Black $^{3,4} \cdot$ Paola Giunti $^{3,4} \cdot$ Doris-Eva Bamiou $^{1,2,5}$
}

Accepted: 25 January 2021 / Published online: 18 February 2021

(C) The Author(s) 2021

\begin{abstract}
Auditory neural impairment is a key clinical feature of Friedreich's Ataxia (FRDA). We aimed to characterize the phenotypical spectrum of the auditory impairment in FRDA in order to facilitate early identification and timely management of auditory impairment in FRDA patients and to explore the relationship between the severity of auditory impairment with genetic variables (the expansion size of GAA trinucleotide repeats, GAA1 and GAA2), when controlled for variables such as disease duration, severity of the disease and cognitive status. Twenty-seven patients with genetically confirmed FRDA underwent baseline audiological assessment (pure-tone audiometry, otoacoustic emissions, auditory brainstem response). Twenty of these patients had additional psychophysical auditory processing evaluation including an auditory temporal processing test (gaps in noise test) and a binaural speech perception test that assesses spatial processing (Listening in Spatialized Noise-Sentences Test). Auditory spatial and auditory temporal processing ability were significantly associated with the repeat length of GAA1. Patients with GAA1 greater than 500 repeats had more severe auditory temporal and spatial processing deficits, leading to poorer speech perception. Furthermore, the spatial processing ability was strongly correlated with the Montreal Cognitive Assessment (MoCA) score. To our knowledge, this is the first study to demonstrate an association between genotype and auditory spatial processing phenotype in patients with FRDA. Auditory temporal processing, neural sound conduction, spatial processing and speech perception were more severely affected in patients with GAA1 greater than 500 repeats. The results of our study may indicate that auditory deprivation plays a role in the development of mild cognitive impairment in FRDA patients.
\end{abstract}

Keywords Friedreich's ataxia, $\cdot$ Cognition, $\cdot$ Hearing, $\cdot$ Auditory processing, $\cdot$ Phenotype, $\cdot$ GAA1

Paola Giunti and Doris-Eva Bamiou are joint last authors

Nehzat Koohi

n.koohi@ucl.ac.uk

Paola Giunti

p.giunti@ucl.ac.uk

Doris-Eva Bamiou

d.bamiou@ucl.ac.uk

1 The Ear Institute, University College London, London WC1X 8EE, UK

2 Neuro-otology Department, University College London Hospitals, London WC1E 6DG, UK

3 Department of Clinical and Movement Neurosciences, Institute of Neurology, University College London, London WC1N 3BG, UK

4 Ataxia Centre, National Hospital for Neurology and Neurosurgery, University College London Hospitals, London WC1N 3BG, UK

5 Biomedical Research Centre, National Institute for Health Research, London WC1E 6DG, UK

\section{Introduction}

Friedreich's Ataxia (FRDA) is the most frequent autosomal recessive inherited ataxia caused by mutations in the $F X N$ gene. An expansion of a homozygous or heterozygous GAA trinucleotide repeat in intron 1 of the gene, which results in deficiency of the mitochondrial protein frataxin, is present in more than $96 \%$ of mutant alleles [1]. Cardinal features of FRDA are ataxia of both trunk and limbs along with dysarthria, global areflexia, deep sensory loss and pyramidal signs [1]. Sensorineural hearing loss as revealed in a pure-tone audiogram is seen in only 8 to $13 \%$ of FRDA patients but other types of hearing impairment, such as difficulty understanding speech in background noise, are present in more than $90 \%$ of these patients [1-3]. Preneural auditory responses from the cochlear outer hair cells are typically normal in more than $90 \%$ of the patients [4]. Most of these individuals show abnormalities in auditory neural and brainstem responses as a result of axonopathy in the eighth nerve and auditory brainstem, termed as "auditory neuropathy," [4-6] which is 
the cause of listening difficulties $[4,7,8]$. Patients with auditory neuropathy struggle to understand speech when background noise is present $[9,10]$ and show impaired ability to selectively attend to a particular voice based on its location [7].

There is phenotypic variability in FRDA that may, to some extent, be explained by the molecular mechanism underlying the disease. The expansion size of the number of GAA trinucleotide repeats on the shorter allele (hereafter referred to as GAA1) in each pair inversely correlates with age at onset and directly with the presence of diabetes mellitus, cardiomyopathy and severity of peripheral sensory neuropathy $[1,5$, 11-13]. In this connection, the size of GAA1 is more significant than the size of the larger allele (GAA2). There is also auditory phenotypic variability (see Table 1 for a summary of studies) but only a few studies attempted to correlate the size of GAA1 with the severity of auditory impairment. Apart from one study conducted by Rance et al. [4], all showed no correlation between the size of GAA1 and the severity of auditory impairment (Table 1). Rance's study is the single study [4] that reported a significant correlation between an auditory temporal processing test (of amplitude modulation detection, i.e. the ability to detect changes in frequency) and GAA1. Other studies reported no correlation between the repeat length of GAA1 and the severity of auditory processing impairment as reflected by neurophysiology (auditory brainstem evoked responses) or other psychoacoustic tests including speech in noise. As FRDA is a rare disease, these studies had small samples and almost all employed a fairly limited set of tests in order to assess the auditory impairment in FRDA in detail and its correlation with the genotype. Identifying and understanding such associations would be of benefit in the search of biomarkers for this disease.

Our main aim was to characterize the phenotypical spectrum of the auditory sensitivity and auditory processing impairment in FRDA in more detail in order to explore further if the auditory phenotypes correlate with genetic and clinical variables such as GAA1, GAA2, disease duration, severity of the disease and cognitive status. Our exploratory aim was to measure the relationship between patients' auditory processing and cognitive status as one might affect the other $[17,18]$.

\section{Methods}

\section{Standard Protocol Approvals, Registrations and Patient Consents}

This study was registered and conducted as an audit at the University College London Hospitals (UCLH). All procedures were conducted as part of routine clinical care. The study was performed under the ethical guidelines issued by our institution, with written informed consent obtained from all participants.

\section{Participants and Settings}

Our neuro-otology clinic is part of The Ataxia Centre's multidisciplinary team at UCLH. Between 2016 and 2020, clinicians working in the Ataxia Centre referred twenty-seven FRDA patients, who carried GAA expansions in intron 1 of FXN gene, to the Neuro-otology Department of UCLH for hearing assessment. From this sample, all patients had puretone audiometry and otoacoustic emissions; 26 patients had auditory brainstem response; 21 speech in quiet test; 18 speech in babble test; 20 listening in spatialized noise test; and 21 gaps in noise test. Diagnostic details for each individual with FRDA were collected (Table 2). Age at assessment ranged from 17 to 58 years ( $32.8 \pm 11.4$ years). The number of GAA1 and GAA2 repeats ranged from 100 to 1050 (684 \pm $258)$ and 220 to $1680(948 \pm 261)$, respectively. The disease duration, which was calculated by subtracting the age at onset from the age of the patient at assessment, ranged from 7 to 44 years $(22.6 \pm 10.5)$. Patients were classified into three groups, those with GAA1 repeats over 700, those between 500 and 700 and those with repeats under 500 [1]. No significant statistical differences were found in disease duration, age at assessment, age at onset and Scale for the Assessment and Rating of Ataxia (SARA) scores between the three GAA groups.

\section{Baseline Audiological Assessment}

We collected information about the patients' hearing status. After otoscopy and wax removal, patients were tested in a sound-treated booth with the following test procedures as per the standard protocol of the clinic and in accordance with test recommendations by the British Society of Audiology [19].

- Pure-tone audiometry (PTA) - PTA was tested at frequencies $250,500,1000,2000,4000$ and $8000 \mathrm{~Hz}$ to determine the hearing sensitivity levels. The air-conduction PTAs were calculated using two different formulas and rounded to the nearest whole number: pure-tone average $(250,500$, $1000,2000,4000$ and $8000 \mathrm{~Hz}$ ) as per BSA [19] protocol and low frequency average ( 250 and $500 \mathrm{~Hz}$ ). The degree of hearing loss was determined as per BSA protocol [19].

- Otoacoustic emissions (OAEs), Transient-evoked and distortion product otoacoustic emissions (TEOAEs and DPOAEs), were used to measure the functioning of the cochlear outer hair cells. Normal response was considered the finding of overall TEOAEs amplitude $+12 \mathrm{~dB}$ or amplitude of $+6 \mathrm{~dB}$ in at least three adjacent frequency bands. 
Table 1 Audiological abnormalities in FRDA individuals

\begin{tabular}{|c|c|c|c|c|c|c|}
\hline Study & Audiological tests & Hearing impairment & $\begin{array}{l}\text { GAA correlation } \\
\text { performed? }\end{array}$ & $\begin{array}{l}\text { No of } \\
\text { patients }\end{array}$ & $\begin{array}{l}\text { Age } \\
\text { range }\end{array}$ & $\begin{array}{l}\text { GAA } \\
\text { range }\end{array}$ \\
\hline $\begin{array}{l}\text { Jabbari et al., } 1983 \\
\quad[5]\end{array}$ & ABR, ART, Tymp & $100 \%$ abnormal ABR & No & 5 & & - \\
\hline Durr et al., 1996 [1] & PTA, ABR & $\begin{array}{l}13 \% \text { abnormal PTA, } 61 \% \text { abnormal } \\
\text { ABR }\end{array}$ & $\begin{array}{l}\text { Yes (no correlation was } \\
\text { found) }\end{array}$ & $\begin{array}{r}140 \text { (only } 69 \\
\text { had ABR) }\end{array}$ & $7-77$ & $120-1700$ \\
\hline $\begin{array}{l}\text { Santoro et al., } 2000 \\
\quad[14]\end{array}$ & $\mathrm{ABR}$ & - & $\begin{array}{l}\text { Yes (no correlation was } \\
\text { found) }\end{array}$ & 24 & $9-43$ & $200-1093$ \\
\hline $\begin{array}{l}\text { Rance et al., } 2008 \\
\quad[10]\end{array}$ & $\begin{array}{l}\text { PTA, ABR, ART, } \\
\text { speech in noise test }\end{array}$ & $\begin{array}{l}\text { 30\% abnormal ABR and ART, } 90 \% \\
\text { abnormal speech in noise }\end{array}$ & $\begin{array}{l}\text { Yes, (no correlation was } \\
\text { found) }\end{array}$ & 10 & $8-28$ & $447-780$ \\
\hline Rance et al., 2010 [4] & $\begin{array}{l}\text { PTA, OAEs, ABR, } \\
\text { temporal } \\
\text { processing test }\end{array}$ & $\begin{array}{l}64 \% \text { with temporal processing deficit, } \\
50 \% \text { abnormal ABR, }\end{array}$ & $\begin{array}{l}\text { Yes, (significant } \\
\text { correlation was found } \\
\text { only between } \\
\text { amplitude modulation } \\
\text { detection and GAA1) }\end{array}$ & 14 & $16-52$ & $447-1099$ \\
\hline Rance et al., 2012 [8] & PTA, ABR, LiSN-S & $\begin{array}{l}52 \% \text { abnormal ABR, } 22 \% \text { abnormal } \\
\text { PTA ( } 4 \text { freq. average) }\end{array}$ & $\begin{array}{l}\text { Yes (no correlation was } \\
\text { found between LiSN-S } \\
\text { subscores and GAA1) }\end{array}$ & 23 & $9-55$ & $447-1298$ \\
\hline $\begin{array}{l}\text { Zeigelboim et al., } \\
\quad 2018 \text { [15] }\end{array}$ & $\begin{array}{l}\text { PTA, ABR, } \\
\text { Immittance }\end{array}$ & $\begin{array}{c}43 \% \text { abnormal PTA, } 57 \% \text { abnormal } \\
\text { ABR, } 50 \% \text { abnormal immittance }\end{array}$ & No & 30 & $6-72$ & - \\
\hline $\begin{array}{l}\text { Giroudet et al., } 2018 \\
\quad[16]\end{array}$ & $\begin{array}{l}\text { PTA, OAEs, standard } \\
\text { ABR, split ABR, } \\
\text { speech in noise }\end{array}$ & $\begin{array}{l}\text { 24\% abnormal PTA, 75\% abnormal } \\
\text { speech in noise, } 92 \% \text { abnormal } \\
\text { standard ABR, 38\% abnormal split } \\
\text { ABR }\end{array}$ & No & 37 & $12-63$ & - \\
\hline $\begin{array}{l}\text { Koohi et al., (present } \\
\text { study) }\end{array}$ & $\begin{array}{l}\text { PTA, OAEs, ABR, } \\
\text { SiQ, SiB, LiSN-S, } \\
\text { GIN }\end{array}$ & $\begin{array}{l}\text { 45\% abnormal PTA, } 15 \% \text { abnormal } \\
\text { OAEs, } 77 \% \text { abnormal ABR, } 77 \% \\
\text { abnormal SiQ, } 100 \% \text { abnormal SiB, } \\
\text { 95\% abnormal LiSN-S, } 77 \% \\
\text { abnormal GIN }\end{array}$ & Yes & 27 & $17-58$ & $100-1050$ \\
\hline
\end{tabular}

ABR, auditory brainstem response; PTA, pure-tone audiometry; Tymp, tympanometry; ART, acoustic reflex threshold; OAEs, otoacoustic emissions; GIN, gaps in noise; LiSN-S, Listening in Spatialized; Noise Sentences Test SiB, speech in babble test; SiQ, speech in quiet

- Auditory-evoked brainstem responses (ABRs) - ABRs are sensitive to auditory nerve and brainstem function abnormalities and thus useful in evaluating undetected damage to the auditory system [20,21]. Monaural alternating click stimuli of $100 \mathrm{msec}$ were presented at a rate of 11.1/ sec via headphones. The electrical activity was amplified and filtered (range: 100-3000 Hz). A total of 1000 stimuli were given, with a mean window of $10 \mathrm{msec}$.

- Speech-in-quite test- the Arthur Boothroyd speech recognition test [22] was performed to assess speech intelligibility in quiet.

\section{Auditory Processing Assessment}

Auditory processing disorder (APD) is defined as a specific deficit in the processing of auditory information along the central auditory nervous system, including bottom-up and top-down neural connectivity [23]. Psychoacoustic test batteries of speech and nonspeech stimuli are used to assess auditory processing in the central auditory nervous system. Temporal resolution (auditory temporal processing) is dependent on neural synchrony [24] and reported to be impaired in auditory neuropathy and FRDA patients [4-6]. Speech in noise test (auditory stream segregation) is also impaired in individuals with auditory neuropathy, FRDA [8] and brainstem pathology [25]. Thus, a clinical temporal resolution test, gaps in noise test and a speech understanding in noise test, Listening in Spatialized Noise-Sentences Test, were utilized to assess the auditory processing of the FRDA patients in our cohort.

Gaps in Noise Test (GIN) - GIN measures temporal resolution, which is the ability to follow rapid changes in the envelope of an auditory stimulus over time [26]. The GIN provides an estimate of threshold (shortest gap identified), a total percentage correct responses score and an estimate of attention levels.

Listening in Spatialized Noise-Sentences Test (LiSN-S) the LiSN-S is a clinical speech understanding in noise test which measures the listener's capacity to segregate a target speech signal from a competing speech noise and assesses binaural speech perception and the ability to integrate interaural timing cues in particular [27]. The test is administered under headphones, but a three-dimensional auditory environment is created by synthesizing the stimuli with headrelated transfer functions [27]. Speech reception threshold (the 
Table 2 FRDA patient details

\begin{tabular}{|c|c|c|c|c|c|c|}
\hline Subject & $\begin{array}{l}\text { Age at assessment } \\
\text { (years), gender }\end{array}$ & $\begin{array}{l}\text { Age at onset } \\
\text { (years) }\end{array}$ & $\begin{array}{l}\text { Disease duration } \\
\text { (years) }\end{array}$ & GAA1 & GAA2 & SARA \\
\hline FRDA1* & $18, \mathrm{~F}$ & 11 & 7 & 100 & 650 & 12 \\
\hline FRDA2* & $36, \mathrm{~F}$ & 2 & 34 & 150 & 850 & 26 \\
\hline FRDA3* & $17, \mathrm{M}$ & 4 & 13 & 220 & 220 & 27.5 \\
\hline FRDA4* & $48, \mathrm{~F}$ & 17 & 31 & 400 & 834 & 30 \\
\hline FRDA5* & $42, \mathrm{~F}$ & 17 & 25 & 400 & 800 & 23 \\
\hline FRDA6* & $45, \mathrm{~F}$ & 15 & 30 & 467 & 967 & 26 \\
\hline FRDA $7 * *$ & $37, \mathrm{~F}$ & 19 & 28 & 567 & 900 & 30 \\
\hline FRDA8** & $40, \mathrm{M}$ & 20 & 20 & 567 & 967 & 20 \\
\hline FRDA9** & $21, \mathrm{M}$ & 6 & 15 & 580 & 745 & 23 \\
\hline FRDA10** & $30, \mathrm{M}$ & 11 & 19 & 634 & 767 & 30 \\
\hline FRDA11** & $58, \mathrm{~F}$ & 15 & 43 & 683 & 983 & 40 \\
\hline FRDA12** & $18, \mathrm{M}$ & 5 & 15 & 700 & 850 & 16 \\
\hline FRDA13**** & $40, \mathrm{~F}$ & 8 & 32 & 720 & 920 & 39 \\
\hline FRDA14**** & $28, \mathrm{~F}$ & 5 & 23 & 745 & 945 & 29 \\
\hline FRDA15**** & $34, \mathrm{M}$ & 8 & 26 & 785 & 785 & 30 \\
\hline FRDA16**** & $26, \mathrm{M}$ & 8 & 18 & 800 & 867 & 26.6 \\
\hline FRDA17 $* * * *$ & $51, \mathrm{~F}$ & 7 & 44 & 820 & 820 & 37 \\
\hline FRDA18**** & $24, \mathrm{~F}$ & 12 & 12 & 834 & 1034 & 17 \\
\hline FRDA19**** & $41, \mathrm{M}$ & 7 & 34 & 834 & 1167 & 38 \\
\hline FRDA20**** & $20, \mathrm{M}$ & 8 & 12 & 850 & 850 & 20.5 \\
\hline FRDA21**** & $22, \mathrm{M}$ & 15 & 7 & 850 & 1200 & 21.5 \\
\hline FRDA22 $* * *$ & $30, \mathrm{M}$ & 11 & 19 & 850 & 1350 & 36 \\
\hline FRDA23**** & $50, \mathrm{M}$ & 13 & 37 & 850 & 1050 & 28 \\
\hline FRDA24**** & $31, \mathrm{~F}$ & 22 & 9 & 1000 & 1000 & 21 \\
\hline FRDA25*** & $25, \mathrm{~F}$ & 7 & 18 & 1000 & 1200 & 40 \\
\hline FRDA26**** & $31, \mathrm{~F}$ & 3 & 28 & 1020 & 1220 & 40 \\
\hline FRDA27**** & $21, \mathrm{M}$ & 5 & 16 & 1050 & 1680 & 29 \\
\hline
\end{tabular}

*, GAA1 < 500; **, GAA1 500-700; ***GAA1 > 700

signal-to-noise ratio required for the listener to identify $50 \%$ of the words in target sentences) is established in four conditions which vary in terms of the location of the noise source $(0$ versus 90 azimuth) and vocal quality of the speaker (same or different talker used to produce the target and background signals). Two "advantage" measures representing the $\mathrm{dB}$ benefit afforded by "spatial" or "talker" cues are also calculated. For the LiSN-S, z-scores for each of the four conditions and the spatial advantage measure were generated according to age-specific normative data [28].

\section{Hearing Questionnaires}

The Speech, Spatial and Qualities of Hearing (SSQ) questionnaire measures a range of hearing disabilities across several hearing functions that are presumed to be served to advantage by the binaural system [29]. Bamiou et al. [30] found a correlation between the GIN test and SSQ scores indicating that temporal processing deficits may play an important role in clinical presentation. The SSQ consists of 49 questions organized into 3 sub-tests (Speech, 14 items; Spatial, 17 items; Quality, 18 items). Each item is rated from 0 (inability to hear) to 10 (perfect hearing). This questionnaire is completed in the clinic by the patient with the help of his/her communication partner as needed.

\section{The Montreal Cognitive Assessment (MoCA)}

When referring to auditory perception, one important everyday skill is speech perception which is known to be related to cognition [31, 32]. Specifically, the performance on speech perception is linked with working memory measurements [33]. However, the accuracy of some cognitive measurements may be negatively influenced when the auditory sensory system is not functioning properly $[17,18,34]$. Research in FRDA suggests some impaired cognitive skills such as 
attention and working memory in these patients [35, 36], both of which are confounding factors in the central auditory processing function. In order to control for confounding cognitive factors, we collected some information on the cognitive status of patients using the Montreal Cognitive Assessment (MoCA), which is a brief screening test that assesses a wide range of cognitive functions sensitive to mild cognitive impairment. MoCA [37] that includes sections on visuospatial/ executive function, naming, attention, language, abstraction, memory and orientation to time and place (30-point test cutoff of $<26 / 30$ for mild cognitive impairment) was conducted by a qualified specialist at the Ataxia Centre at UCLH as part of routine clinical assessment.

\section{Statistical Analysis}

Data were analysed using the Statistical Package for the Social Science (IBM SPSS Statistics for Windows, Version 22.0; IBM Corp., Armonk, NY). Differences in mean size of the GAA1 and GAA2 repeats between groups categorized on the presence of audiological abnormality (LiSN-S, normal vs. abnormal; ABR, normal vs. abnormal; GIN, normal vs. abnormal; PTA, normal vs. abnormal) were assessed for statistical significance by two-tailed $t$ test. A $p$ value $<0.05$ was taken to indicate statistical significance. A series of hierarchical multiple linear regression analysis was used to explore associations between LiSN-S scores, GIN thresholds, subscores of SSQ questionnaire and a range of genetic variables including the repeat length of GAA1 and GAA2. The controlled factors were disease duration, MoCA and SARA scores. A partial correlation was run to determine the relationship between LiSN-S scores, GIN thresholds, subscores of SSQ questionnaire and MoCA score whilst controlling for GAA1, disease duration and SARA scores. One-way analysis of variance (ANOVA) and post hoc Bonferroni were used to compare mean GIN thresholds in patients with GAA1 less than 500, 500-700 and more than 700 .

\section{Results}

\section{Baseline Audiological Assessment and Association Between GAA Repeat Sizes and the Presence of Audiological Abnormalities}

Hearing assessment of six FRDA patients with GAA1 repeats less than 500 revealed no/mild hearing impairment (patients 1-6). Mild/moderate hearing impairment was observed in 21 patients with GAA1 repeat lengths of more than 500 (patients 7-27). The association between the size of GAA1 and GAA2 repeats and the presence or absence of audiological abnormalities were assessed. The mean allele sizes were compared in those with audiological abnormalities and those without. A significant association with GAA1 size is observed for PTA average $(P=0.02)$, ABR $(P=0.000), \mathrm{SiQ}(P=0.012)$, LiSN$\mathrm{S}(P=0.04)$ and $\mathrm{GIN}(P=0.000)$. The GAA2 was associated with the presence of abnormality in GIN test. The results are summarized in Tables 3 and 4.

\section{Binaural Speech Perception-LiSN-S Test}

Summary measures (z-scores) of the five subscores of LiSN-S for the three GAA groups are shown in Fig. 1a. A series of hierarchal multiple linear regressions were calculated to predict LiSN-S scores on GAA1 and GAA2 repeats after controlling for disease duration, SARA and MoCA scores. Preliminary analyses were conducted to ensure no violation of the assumptions of normality and collinearity. No association was found between findings for two of the listening conditions (talker and total advantage) and GAA1 and GAA2 repeats size. No association was found between findings for all of the listening conditions and GAA2 repeats size. However, a hierarchal multiple linear regression analysis to predict LiSN-S spatial advantage score from GAA1 repeats showed a significant equation $(\mathrm{F}(4,16)=6.9, p<0.05)$, with $\mathrm{R}$ squared of 0.7 and an adjusted $\mathrm{R}$ squared of 0.6 . A significant association was also found between the LiSN-S high-cue subscore $(\mathrm{F}(4,16)=4.7, p<0.05)$, with $\mathrm{R}$ squared of 0.6 and an adjusted $\mathrm{R}$ squared of 0.5 , as well as low-cue subscore $(\mathrm{F}(4$, $16)=3.5, p<0.05$ ), with $\mathrm{R}$ squared of 0.5 and an adjusted $\mathrm{R}$ squared of 0.4. Figure 2a shows spatial advantage, disease duration, SARA score, GAA1 and GAA2 repeats findings for each FRDA patient.

\section{Temporal Resolution-Gaps in Noise}

GIN results were within normal limits in patients with GAA1 less than 500 (Fig. 1b). A hierarchal multiple linear regression was calculated to predict the effect of GAA1 and GAA2 repeats on GIN thresholds when controlled for disease duration, SARA and MoCA scores. A significant equation was found $(\mathrm{F}(5,16)=7.5, p<0.05)$, with $\mathrm{R}$ squared of 0.71 and an adjusted $\mathrm{R}$ squared of 0.62 . Similar to spatial advantage score of LiSN-S, only GAA1 added statistically significantly to the prediction, $p<0.05$. Figure $2 \mathrm{~b}$ shows GIN threshold, disease duration, SARA score, GAA1 and GAA2 repeats findings for each FRDA patient.

There was a statistically significant difference between the right GIN thresholds of three GAA1 groups as determined by one-way ANOVA $(\mathrm{F}(2,16)=19.96, p<0.001)$ as well as left GIN thresholds $(\mathrm{F}(2,16)=11.42, p<0.001)$. A Bonferroni post hoc test revealed that the GIN threshold in patients with GAA1 less than 500 was statistically significantly lower compared to those patients with GAA1 500-700 and GAA1 more than 700 . There was no statistically significant difference 
Table 3 Distribution of audiological findings in FRDA patients

\begin{tabular}{|c|c|c|c|c|c|c|}
\hline Audiological presentation & No. of patients $N(27)$ & $\begin{array}{l}\text { Age } \\
\text { (years old) }\end{array}$ & GAA1 & GAA2 & $\begin{array}{l}p \text { value } \\
\text { GAA1 }\end{array}$ & $\begin{array}{l}p \text { value } \\
\text { GAA2 }\end{array}$ \\
\hline \multicolumn{7}{|l|}{ PTA (BSA average) } \\
\hline Normal & $15 / 27(55 \%)$ & 28.9 & 583 & 866 & $0.02 *$ & 0.17 \\
\hline $\begin{array}{l}\text { Abnormal } \\
\text { PTA (LF average) }\end{array}$ & $12 / 27(45 \%)$ & 37.5 & 810 & 1026 & & \\
\hline Normal & $15 / 27(55 \%)$ & 28.5 & 601 & 865 & 0.14 & 0.14 \\
\hline $\begin{array}{l}\text { Abnormal } \\
\text { OAEs }\end{array}$ & $12 / 27(45 \%)$ & 36.3 & 750 & 1015 & & \\
\hline Present & $23 / 27(85 \%)$ & 31.1 & 661 & 922 & 0.27 & 0.21 \\
\hline $\begin{array}{l}\text { Absent } \\
\text { ABR }\end{array}$ & $4 / 27(15 \%)$ & 42.5 & 818 & 1100 & & \\
\hline Normal & $6 / 26(23 \%)$ & 34.3 & 289 & 720 & $0.000 *$ & 0.45 \\
\hline $\begin{array}{l}\text { Abnormal } \\
\text { SiQ }\end{array}$ & $20 / 26(77 \%)$ & 31.1 & 802 & 1015 & & \\
\hline Normal & $5 / 22(23 \%)$ & 32.8 & 380 & 796 & $0.012 *$ & 0.28 \\
\hline $\begin{array}{l}\text { Abnormal } \\
\mathrm{SiB}\end{array}$ & $17 / 22(77 \%)$ & 31.1 & 724 & 950 & & \\
\hline Normal & $0 / 18(0 \%)$ & 27.6 & 687 & 925 & - & - \\
\hline $\begin{array}{l}\text { Abnormal } \\
\text { LiSN-S }\end{array}$ & $18 / 18(100 \%)$ & & & & & \\
\hline Normal/Mild SPD & $3 / 20(15 \%)$ & 35.6 & 340 & 618 & $0.04 *$ & 0.06 \\
\hline $\begin{array}{l}\text { SPD } \\
\text { GIN }\end{array}$ & $17 / 20(85 \%)$ & 29.2 & 719 & 976 & & \\
\hline Normal & $7 / 21(33 \%)$ & 32.3 & 369 & 738 & $0.000^{*}$ & $0.04 *$ \\
\hline Abnormal & $14 / 21(77 \%)$ & 29.6 & 722 & 1010 & & \\
\hline
\end{tabular}

*denotes significance. LF, low frequency; BSA, British Society of Audiology; PTA, pure-tone audiometry; OAEs, otoacoustic emissions; $\mathrm{ABR}$, auditory-evoked brainstem responses; $\mathrm{SiQ}$, speech in quiet; $\mathrm{SiB}$, speech in babble; LiSN-S, listening in spatialized noise sentences; GIN, gaps in noise test; Ab, abnormal between those groups with GAA1 500-700 and GAA1 more than 700 .

\section{Patient-Reported Hearing Difficulties-SSQ Questionnaire}

A series of hierarchical multiple linear regressions were calculated with SSQ questionnaire subscores as the dependant variables. SARA score, disease duration and MoCA scores were entered at the stage one of the regression. The genetic variables (GAA1 and GAA2) were entered at stage two and auditory processing variables (GIN and spatial subscore of LiSN-S) at stage three. The auditory processing variables statistically significantly predicted the speech subscore, $F(7,13)$ $=6.1, p<0.05$, with $\mathrm{R}$ squared of 0.88 and an adjusted $\mathrm{R}$ squared of 0.73 . Interestingly, for the spatial subscore, as well as the auditory processing variables, the GAA1 added statistically significantly to the prediction, $F(7,13)=4.3, p<0.05$, with $\mathrm{R}$ squared of 0.83 and an adjusted $\mathrm{R}$ squared of 0.64 . There was no relation between the Quality and total subscores, disease duration, SARA and MoCA scores, auditory processing variables, GAA1 and GAA2 repeats, $p>0.05$.

\section{Cognitive Assessment-MoCA}

To explore the relationship between patients' cognitive status and speech in noise performance, a partial correlation was run to determine the relationship between the subscores of LiSN-S test and MoCA score whilst controlling for GAA1, SARA score and disease duration. There was a strong, partial correlation between the spatial advantage subscore of LiSN-S test $(-4.4 \pm 1.7)$ and MoCA score $(25.6 \pm 3.5)$ whilst controlling for GAA1 (638.04 \pm 268.37$)$, SARA score $(24.83 \pm 5.75)$ and disease duration $(19.52 \pm 8.67)$, which was statistically significant, $r(12)=0.62, N=17, p=0.018$. A partial correlation was also found between the high-cue subscore of the LiSN-S test and MoCA score, $r(12)=0.67, N=17, p=0.009$.

A partial correlation was run to determine the relationship between the GIN thresholds and MoCA score whilst controlling for GAA1, SARA score and disease duration. There was no partial correlation $(r(12)=0.13, N=17, p=0.67)$ between 


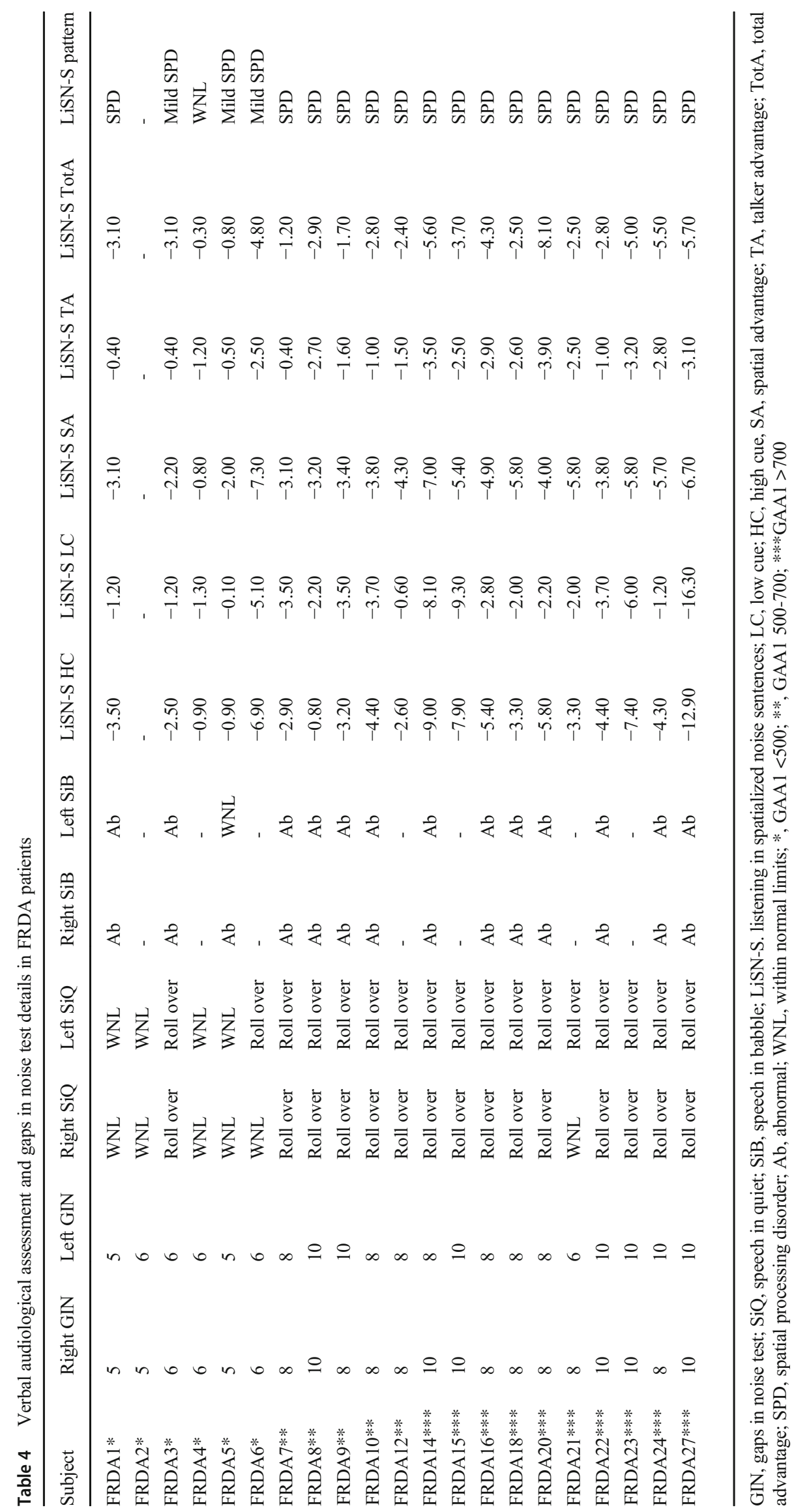


Fig. 1 Speech reception thresholds for five subscores of LiSN-S and GIN results in FRDA patients. a Normal range is shaded in green. Blue line depicts the mean LiSN-S subscores for patients with GAA1 repeats less than 500; red line for GAA1 500 to 700; and green line GAA1 more than 500 . b Normal range is below the dotted line. GIN, gaps in noise test a

\section{Variance from mean}

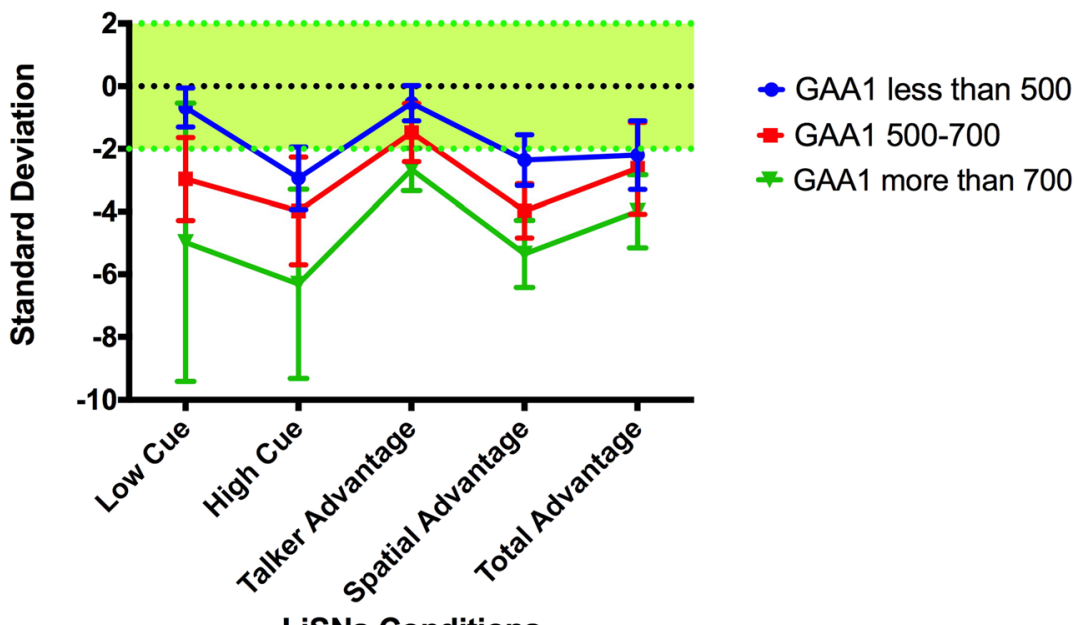

LiSNs Conditions

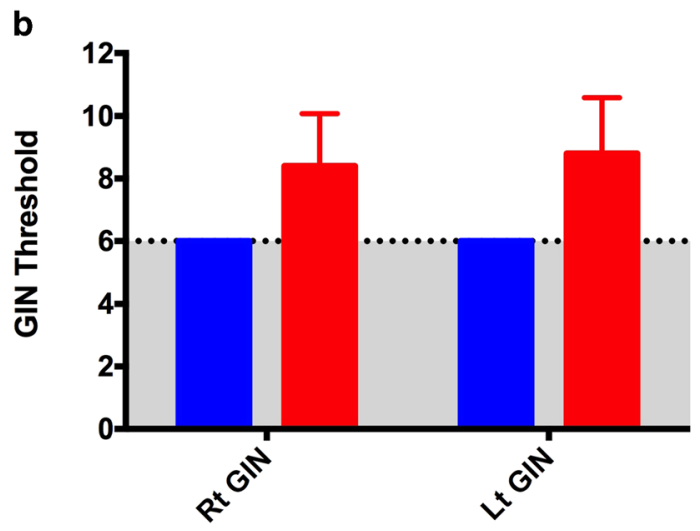

GAA1 less than 500

GAA1 more than 500 the GIN thresholds $(7.86 \pm 1.77)$ and MoCA score $(25.6 \pm 3.5)$ whilst controlling for GAA1 (638.04 \pm 268.37$)$, SARA score $(24.83 \pm 5.75)$ and disease duration (19.52 \pm 8.67$)$.

No correlation was found between the SSQ subscores and MoCA scores.

\section{Discussion}

The Friedreich's ataxia molecular defect to some extent can explain the phenotypic variability of the disease. Previous research $[11,14]$ prompted us to investigate the relation between the severity of the auditory impairment, as shown on behavioural and physiological audiological assessments, and the expansion size of GAA1 repeats. We demonstrate a more extensive association between genotype and auditory phenotype in patients with FRDA than previously reported. We observed better audiometric thresholds, better ABR waveforms, better auditory temporal, spatial and speech in noise processing in patients with the repeat length of GAA1 less than 500 independent from disease duration, SARA and cognitive function (MoCA scores). Notably, a previous study provided evidence that GAA1 size is the main factor determining the severity of sensory neuropathy [14], consistent with our results. They suggested that the severity of the sensory neuropathy is genetically determined. Ragno et al. [38] described patients with minimal GAA expansion on one allele (ranging from 120 to 156 triplets) and without clinical and electrophysiological signs of sensory neuropathy.

Disrupted neural activity significantly impairs timingrelated perception. This could be due to desynchronization of neural activity due to demyelination of the auditory nerve [39] or disrupted axonal transmission [40]. Previous studies have shown that the auditory neural pathway disruption in FRDA individuals is probably due to axonal degeneration [41, 42] rather than desynchronization. The findings of our study suggest that temporal distortion is mainly observed in FRDA individuals with the GAA1 more than 500. All of our five FRDA patients with GAA1 less than 500 exhibited normal GIN thresholds which was significantly lower than those with GAA1 between 500 and 700 and those more than 700 (Fig. 2b). This severity was not correlated with the repeat length of GAA2 and disease duration. This is the first study 
a
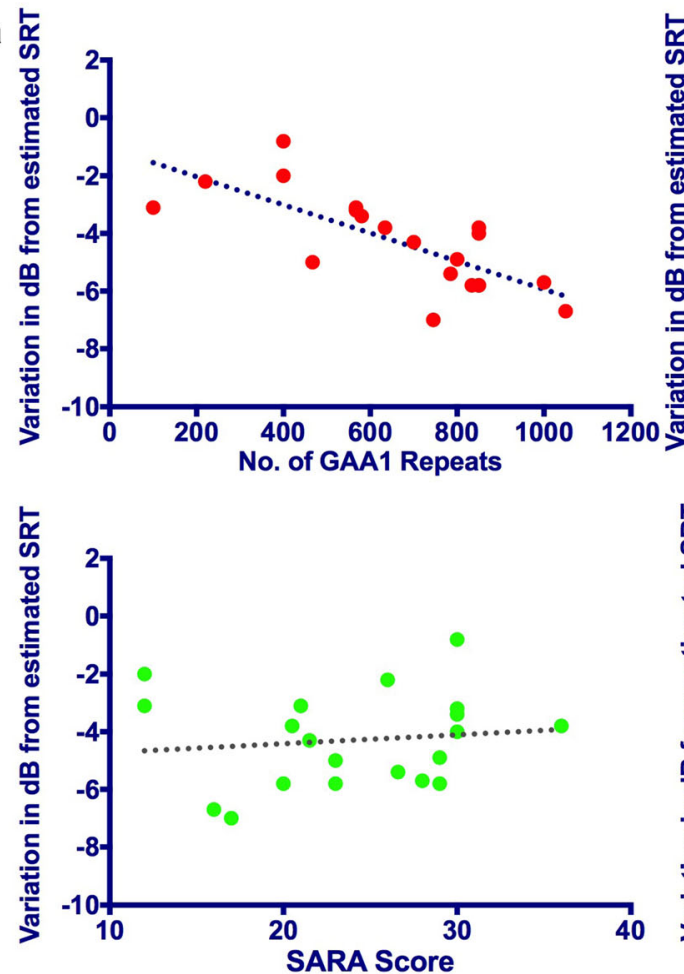

b

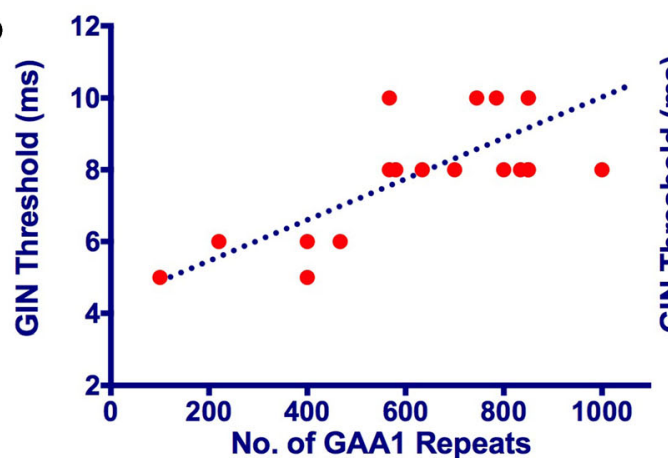

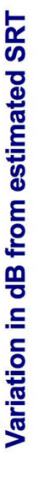

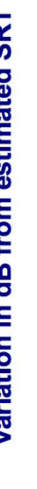
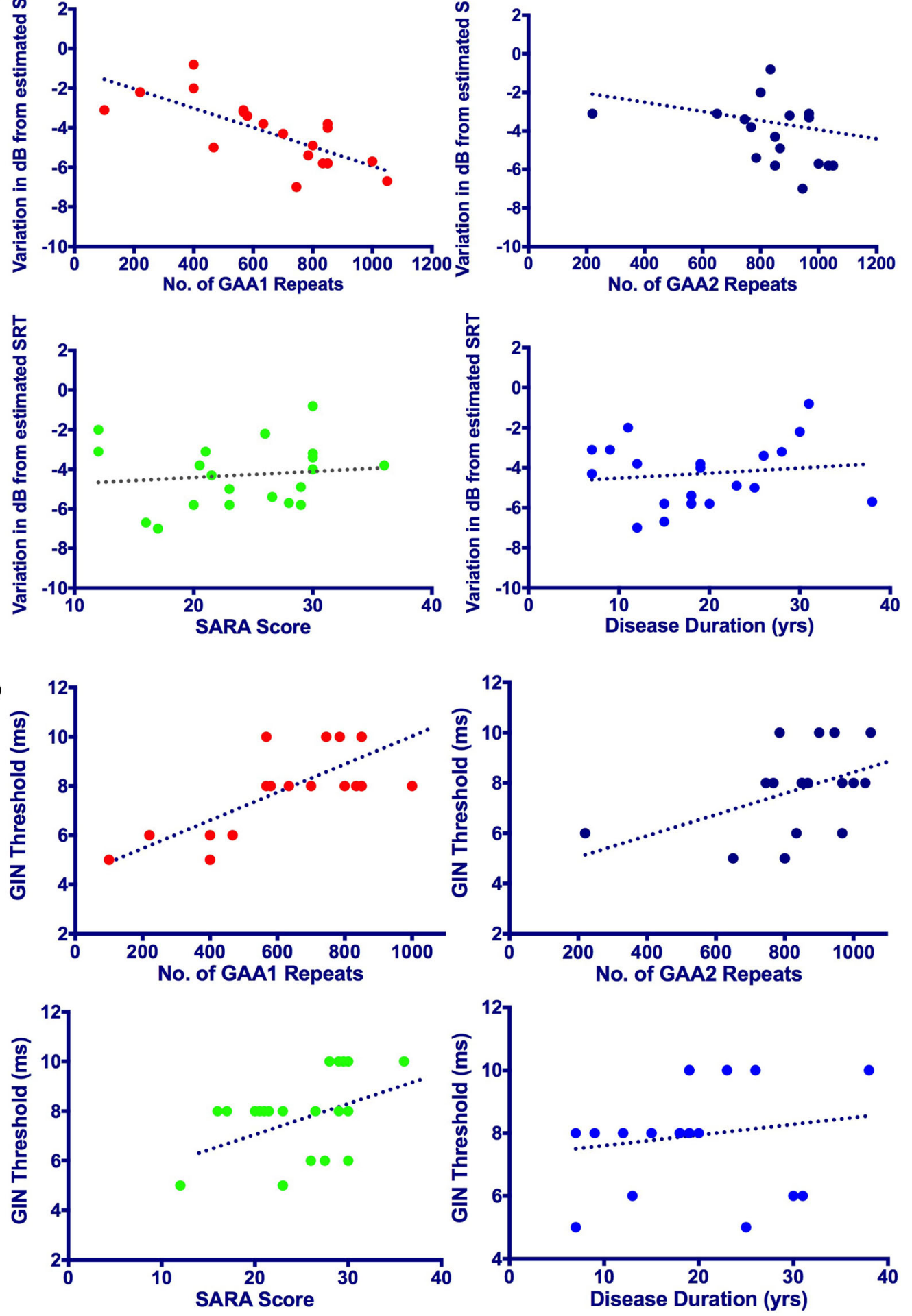

Fig. 2 Relationship between the spatial advantage subscore of LiSN-S and GIN threshold with genetic and clinical variables. a Relationship between the spatial-advantage subscore and GAA1 repeats, GAA2 repeats, SARA score and disease duration findings for each FRDA

to show this relationship between the temporal distortion severity and the repeat length of GAA1 in FRDA patients. Previously, Rance et al. [4] found no relationship between subject. b Relationship between the GIN threshold and GAA1 repeats, GAA2 repeats, SARA score and disease duration findings for each FRDA subject

the repeat length of GAA1 and severity of the impaired temporal processing. One possible reason for this discrepancy is that in that cohort, only one of the 14 FRDA patients had the 
GAA1 less than 500. In addition, different diagnostic tests were used for assessing the auditory processing skills.

Individuals with auditory neuropathy have difficulties integrating the timing cues arriving from the left and right auditory nerves [9]. This impairs the listener's ability to understand speech in the presence of background noise. Rance et al. [8] demonstrated that speech perception in noise for their cohort of 23 FRDA patients was particularly affected in circumstances where binaural processing might have improved perception through spatial segregation. Spatial processing was also related to overall disease severity, as measured by the Friedreich Ataxia Rating Scale (FARS). Confirming their findings, we also observed binaural impaired speech perception in 19 out of 20 FRDA patients. However, the severity of spatial processing impairment was in varying degrees and strongly associated only with the repeat length of GAA1. Unlike Rance's study, we did not find any relationship between any components of the LiSN-S test and overall disease severity. This disparity can be explained by the difference in their cohort of patients in that they only had one patient with the GAA1 less than 500. In addition, we used age-adjusted zscores when calculating the LiSN-S test measures (i.e. $\leq-2.0$ standard deviations from age-normalized data), whilst Rance's study used the signal-to-noise ratio measures; thus, age effects may not have been entirely accounted for.

The structural integrity of grey matter within the cerebral and cerebellar cortices in FRDA patients was investigated in imaging study by Selvadurai et al. [43] Reduced cortical thickness and volume were observed in the prefrontal cortices, insula and temporal poles, all of which are part of the central auditory nervous system (CANS). Notably, a mild spatial processing disorder was observed even in those FRDA patients with normal $A B R$ and those with unilateral auditory neuropathy (FRDA 3-6), which suggests their hearing impairment may also be due to CANS involvement, beyond the axonopathy in the eighth nerve and auditory brainstem, and may index other CANS problems.

\section{Patient-Reported Hearing Difficulties}

The present study set out to determine whether the presence of auditory processing deficit is related to real-life listening difficulty and if this is also related to the repeat length of GAA1. All patients whose GAA1 was more than 500 reported gross functional deficits in a variety of complex listening situations typical of those encountered in everyday life (evident on the speech, spatial and qualities of hearing scale questionnaire). With the exception of FRDA \#6 with the disease duration of 30 years and SARA score of 26 and FRDA \# 1 with the disease duration of 11 years and SARA score of 12 who scored low in the speech subscore of SSQ, the remaining FRDA individuals with GAA1 less than 500 did not report gross difficulties in complex listening conditions. Controlled for the disease duration, SARA and MoCA scores, only the spatial subscale of SSQ was shown to be strongly related to the repeat length of GAA1 as well as auditory processing variables. The speech subscale was only strongly related to the auditory processing variables.

There are limitations in using self-report questionnaires in that it is not clear whether the reports of the FRDA patients were in fact accurate representations of their experiences. However, our findings suggest that the spatial subscore of SSQ questionnaire may be used as a screening tool to identify those patients with auditory processing deficits.

\section{Cognitive Assessment-MoCA}

When the encoding of sound is impaired, greater cognitive resources are required for auditory perceptual processing to the detriment of other cognitive processes such as working memory $[35,36,44]$. There is emerging research to suggest that patients with Friedrich's Ataxia are at risk of cognitive impairment that may impact on these individuals' communication. The findings of our study revealed a strong correlation between the MoCA score and two subscores of the LiSN-S test (spatial advantage and high-cue score). A previous study in children with auditory processing disorder [45] showed no association between the LiSN-S-derived subscores and cognitive processes such as auditory working memory and nonverbal intelligence. Long-term auditory deprivation is thought to impact cognitive performance through the reduction in communication and social activities [46]. The results of our study suggest auditory deprivation may have a role in developing mild cognitive impairment in FRDA patients. One way to delay the synergistic effect of auditory deprivation on cognitive processing is to increase the listener's signal-to-noise ratio (SNR) [47], which acts as a bottom-up strategy. The improved SNR facilitates better auditory processing and enhanced understanding speech in noise, due to a clearer signal, which subsequently leads to reduced cognitive load and better cognitive abilities that can be used for other higher cognitive processes such as attention, memory, emotion and language processing.

\section{Conclusions, Limitations and Future Directions}

In summary, the findings of our study suggest the presence of a more severe auditory processing disorder (APD) in those patients with a GAA1 more than 500 leading to more problems with speech perception in background noise. APD's main symptom is difficulty understanding speech in background noise which is considered one of the most incapacitating elements of auditory impairment as it may cause feelings of isolation and affect relationships. Identifying and understanding the cause of this reported difficulty in FRDA patients is essential for hearing rehabilitation of these individuals. 
Additionally, the auditory processing measures may be candidate auditory biomarkers for FRDA severity and intervention benefit.

Future research should aim to test a larger cohort of FRDA patients, particularly those with GAA1 less than 500. Prospect studies should include auditory phenotyping of the paediatric population in order to characterize and classify the auditory impairments, using a detailed audiological assessment test battery. This would enable specific guides for management and intervention for this patient population.

As well as auditory processing deficits, current research suggests that FRDA patients are at risk of cognitive impairment that may also impact their ability to communicate effectively $[35,36]$. The ear's peripheral auditory systems, the central auditory pathways and cognitive systems in the brain must all work in harmony for successful communication in noisy environments $[32,48]$. In particular, there is a link between speech perception performance and working memory, in which information is stored and manipulated temporarily [33], as well as verbal memory [48, 49]. Thus, as well as impaired hearing, cognitive deficits may make it more difficult to understand speech when background noise is present, with consequent worsening of the ability to communicate. Although studies have demonstrated hearing and cognitive impairment in FRDA patients, none, to the best of our knowledge, have examined both hearing and cognitive impairments and how they act together to influence cognitive, emotional and physical health. There is clearly a need to better understand the associations between the hearing and cognitive impairments in FRDA patients and correlate these results with the repeat length of GAA1. Thus, future research should evaluate the relationship and interaction between the degree of auditory impairment, overall clinical severity of the disease and cognitive function using comprehensive cognitive assessment.

Acknowledgments The authors acknowledge the support of the Deafness and Hearing Problems theme NIHR UCLH Biomedical Research Centre (BRC). We would like to thank our patients and colleagues at the Ataxia Centre and Neuro-otology Departments of the University College London Hospitals.

Author Contributions NK: design and conceptualized study; data collection; analysed and interpreted the data; drafted the manuscript for intellectual content. GT-B: data collection; revised the manuscript for intellectual content. PG: design and conceptualized study; interpreted the data; revised the manuscript for intellectual content. D-EB: design and conceptualized study; interpreted the data; revised the manuscript for intellectual content.

\section{Declarations}

Conflict of Interest The authors declare that they have no conflict of interest.
Open Access This article is licensed under a Creative Commons Attribution 4.0 International License, which permits use, sharing, adaptation, distribution and reproduction in any medium or format, as long as you give appropriate credit to the original author(s) and the source, provide a link to the Creative Commons licence, and indicate if changes were made. The images or other third party material in this article are included in the article's Creative Commons licence, unless indicated otherwise in a credit line to the material. If material is not included in the article's Creative Commons licence and your intended use is not permitted by statutory regulation or exceeds the permitted use, you will need to obtain permission directly from the copyright holder. To view a copy of this licence, visit http://creativecommons.org/licenses/by/4.0/.

\section{References}

1. Dürr A, Cossee M, Agid Y, et al. Clinical and genetic abnormalities in patients with Friedreich's ataxia. N Engl J Med. 1996;335:116975 .

2. Harding A. Friedreich's ataxia: A clinical and genetic study of 90 families with an analysis of early diagnostic criteria and intrafamilial clustering of clinical features. Brain. 1981;104:589 620.

3. Lynch D, Farmer J, Balcer L, et al. Friedreich ataxia: effects of genetic understanding on clinical evaluation and therapy. Arch Neurol. 2002;59:743-7.

4. Rance G, Corben L, Barker E, et al. Auditory perception in individuals with Friedreich's ataxia. Audiol Neurotol. 2010;15:229-40.

5. Jabbari B, Schwartz D, MacNeil D, et al. Early abnormalities of brainstem auditory evoked potentials in Friedreich's ataxia: evidence of primary brainstem dysfunction. Neurology. 1983;33: 1071-4.

6. Santarelli R, Cama E, Pegoraro E, et al. Abnormal cochlear potentials in Friedreich's ataxia point to disordered synchrony of auditory nerve fiber activity. Neurodegener Dis. 2010;15:114-20.

7. Rance G, Corben L, Delatycki M, et al. Auditory processing deficits in children with Friedreich ataxia. J Child Neurol. 2012a;27:1197203.

8. Rance G, Ryan M, Carew P, et al. Binaural speech processing in individuals with auditory neuropathy. Neuroscience. 2012b;13: 227-35.

9. Zeng F. Perceptual consequences of disrupted auditory nerve activity. J Neurophysiol. 2005;93:3050-63.

10. Rance G, Fava R, Baldock H, et al. Speech perception ability in individuals with Friedreich ataxia. Brain. 2008;131:2002-12.

11. Filla A, Michele G. The relationship between trinucleotide (GAA) repeat length and clinical features in Friedreich ataxia. Am J Hum Genet. 1996;59:554-60.

12. Montermini L, Andermann E, Labuda M, et al. The Friedreich ataxia GAA triplet repeat: Premutation and normal alleles. Hum Mol Genet. 1997;6:1261-6.

13. Parkinson M, Boesch S, Nachbauer W, et al. Clinical features of Friedreich's ataxia: classical and atypical phenotypes. J Neurochem. 2013;126(S1):103-17.

14. Santoro L, De Michele G, Perretti A, et al. Relation between trinucleotide GAA repeat length and sensory neuropathy in Friedreich's ataxia. J Neurol Neurosurg Psychiatry. 1999;66:93-6.

15. Zeigelboim B, Teive H, Rosa M, et al. The importance of central auditory evaluation in Friedreich's ataxia. Arq Neuropsi- Quiatr. 2018;76:170-6.

16. Giraudet F, Charles P, Mom T, et al. Rapid exhaustion of auditory neural conduction in a prototypical mitochondrial disease, Friedreich ataxia. Clin Neurophysiol. 2018;129:1121-9. 
17. Iliadou V, Moschopoulos N, Sidiras C, et al. Over-diagnosis of cognitive deficits in psychiatric patients may be the result of not controlling for hearing sensitivity and auditory processing. Psychiatry Clin Neurosci. 2018;72(9):742.

18. Johnson J, Marshall C, Weil R, et al. Hearing and dementia: from ears to brain. Brain. 2020;awaa429

19. British Society of Audiology. Recommended procedure. Boneconduction threshold audiometry with and without masking. In: Br Soc Audiol; 2011.

20. Pillion J, Moser H, Raymond G. Auditory function in adrenomyeloneuropathy. J Neurol Sci. 2008;269:24-9.

21. Jiang Z, Wilkinson A. Relationship between brainstem auditory function during the neonatal period and depressed Apgar score. J Matern Fetal Neonatal Med. 2010;23:973-9.

22. Boothroyd A. Developments in speech audiometry. Br J Audiol. 1968;7:368-8.

23. Iliadou V, Ptok M, Grech H, et al. A European perspective on auditory processing disorder-current knowledge and future research focus. Front Neurol. 2017;21(8):622.

24. Harris K, Dubno J. Age-related deficits in auditory temporal processing: unique contributions of neural dyssynchrony and slowed neuronal processing. Neurobiol Aging. 2017;53:150-8.

25. Hwang Y, Lakshmanan R, Davagnanam I, et al. Brainstem phenotype of cathepsin A-related arteriopathy with strokes and leukoencephalopathy. Neurol Genet. 2017;6:165.

26. Musiek F, Shinn J, Jirsa R, et al. GIN (Gaps-In-Noise) test performance in subjects with confirmed central auditory nervous system involvement. Ear Hear. 2005;26:608-18.

27. Cameron S, Dillon H. Development of the listening in spatialized noise-sentences test (LISN-S). Ear Hear. 2007;28:196-211.

28. Cameron S, Glyde H, Dillon H. Listening in Spatialized NoiseSentences Test (LiSN-S): Normative and retest reliability data for adolescents and adults up to 60 years of age. J Am Acad Audiol. 2011;22:697-709.

29. Gatehouse S, Noble I. The Speech, Spatial and Qualities of Hearing Scale (SSQ). Int J Audiol. 2004;43:85-99.

30. Bamiou D, Iliadou V, Zanchetta S, et al. What can we learn about auditory processing from adult hearing questionnaires? J Am Acad Audiol. 2015;26(10):824-37.

31. Akeroyd M. Are individual differences in speech reception related to individual differences in cognitive ability? A survey of twenty experimental studies with normal and hearing-impaired adults. Int $\mathbf{J}$ Audiol. 2008;47:S53-71.

32. Rönnberg J. Cognition in the hearing impaired and deaf as a bridge between signal and dialogue: a framework and a model. Int J Audiol. 2003;42:S68-76.

33. Arlinger S, Lunner T, Lyxell B, et al. The emergence of cognitive hearing science. Scand J Psychol. 2009;50:371-84.

34. Utoomprurkporn $\mathrm{N}$, Woodall $\mathrm{K}$, Stott $\mathrm{J}$, et al. Int $\mathrm{J}$ Geriatr Psychiatry. 2020;35(9):962-71.
35. Wollmann T, Barroso J, Monton F, et al. Neuropsychological Test Performance of Patients with Friedreich's Ataxia. J Clin Exp Neuropsychol. 2002;24:677-86.

36. Mantovan M, Martinuzzi A, Squarzanti F, et al. Exploring mental status in Friedreich's ataxia: A combined neuropsychological, behavioral and neuroimaging study. Eur J Neurol. 2006;13:827-35.

37. Nasreddine Z, Phillips N, Bédirian V, et al. The montreal cognitive assessment, MoCA: a brief screening tool for mild cognitive impairment. J Am Geriatr Soc. 2005;53:695-9.

38. Ragno M, De Michele G, Cavalcanti F, et al. Broadened Friedreich's ataxia phenotype after gene cloning: minimal GAA expansion causes late onset spastic ataxia. Neurology. 1997;49: 1617-20.

39. Waxman S. Conduction in myelinated, unmyelinated, and demyelinated fibers. Arch Neurol. 1977;34:585-9.

40. Glowatzki E, Fuchs P. Transmitter release at the hair cell ribbon synapse. Nat Neurosci. 2003;5:147-54.

41. Hughes J, Brownell B, Hewer R. The peripheral sensory pathway in Friedreich's ataxia: An examination by light and electron microscopy of the posterior nerve roots, posterior root ganglia, and peripheral sensory nerves in cases of Friedreich's ataxia. Brain. 1968;91: 803-18.

42. Nolano M, Provitera V, Crisci C, et al. Small fibers involvement in Friedreich's ataxia. Ann Neurol. 2001;50:17-25.

43. Selvadurai LP, Harding IH, Corben LA, et al. Cerebral and cerebellar grey matter atrophy in Friedreich ataxia: the IMAGE-FRDA study. J Neurol. 2016;263:2215-23.

44. McCoy S, Tun P, Cox L, et al. Hearing loss and perceptual effort: Downstream effects on older adults' memory for speech. Q J Exp Psychol. 2005;58:22-33.

45. Tomlin D, Dillon H, Sharma M, et al. The impact of auditory processing and cognitive abilities in children. Ear Hear. 2015;36: $527-42$.

46. Dawes P, Emsley R, Cruickshanks K, et al. Hearing loss and cognition: the role of hearing aids, social isolation and depression. PLoS One. 2015;10:e119616.

47. Baran J. Managing auditory processing disorders in adolescents and adults. Semin Hear. 2002;23:327-36.

48. Gatehouse $\mathrm{S}$, Ackroyd M. The effects of cueing temporal and spatial attention on word recognition in a complex listening task in hearing-impaired listeners. Trends Amplif. 2008;12:145-61.

49. Fisher M, Mellon S, Wolkowitz O, et al. Neuroscience-informed auditory training in schizophrenia: A final report of the effects on cognition and serum brain-derived neurotrophic factor. Schizophrenia Res: Cogn. 2016;3:1-7.

Publisher's Note Springer Nature remains neutral with regard to jurisdictional claims in published maps and institutional affiliations. 